\title{
Effect of interface recombination on the efficiency of intracavity double diode structures
}

\author{
Toufik Sadi $^{1}$ (D ) Ivan Radevici ${ }^{1} \cdot$ Pyry Kivisaari $^{1} \cdot$ Alberto Casado $^{1} \cdot$ Jani Oksanen $^{1}$
}

Received: 30 November 2018 / Accepted: 1 June 2019 / Published online: 13 June 2019

(c) The Author(s) 2019

\begin{abstract}
In the past ten years, there has been significant progress in solid-state optical refrigeration, causing a renewed interest in the possibility of electroluminescent cooling (ELC) in light emitting diodes (LEDs). More recently, our work on III-As based intracavity double diode structures (DDSs) indicates that the threshold for ELC can be reached, in practice, at high powers and $300 \mathrm{~K}$ if certain non-radiative recombination mechanisms and photodetector (PD) losses are minimized. The studied DDSs consist of a LED, incorporating a high-quality GaAs active layer, optically coupled to a GaAs p-n homojunction PD. Both the LED and PD are integrated in a single device, offering a unique environment for studying ELC. In this paper, we provide a brief overview of the DDS characteristics and investigate the impact of non-radiative interface recombination on the LED, showing how the choice of the barrier layer materials can suppress this effect. We use experimental characterization techniques to calibrate numerical simulations, coupling the drift-diffusion model for charge transport to a photon transport model. To explore the interface effects, we compare DDSs with either $\mathrm{GaInP} / \mathrm{GaAs}$ or $\mathrm{AlGaAs} / \mathrm{GaAs}$ double heterojunctions. The results suggest that $\mathrm{GaInP}$ barriers allow interface recombination suppression that is sufficient to reach internal cooling in the LED.
\end{abstract}

Keywords Electroluminescent cooling · Light-emitting diodes · III-As semiconductors · $\mathrm{GaInP} / \mathrm{GaAs}$ double heterojunctions $\cdot$ Double diode structures

This article is part of the Topical Collection on Numerical Simulation of Optoelectronic Devices, NUSOD' 18.

Guest edited by Paolo Bardella, Weida Hu, Slawomir Sujecki, Stefan Schulz, Silvano Donati, Angela Traenhardt.

Toufik Sadi

toufik.sadi@aalto.fi

1 Engineered Nanosystems Group, School of Science, Aalto University, P.O. Box 12200,

00076 Aalto, Finland 


\section{Introduction}

The light emitting diode (LED) industry has been thriving for the last 20 years, thanks to new materials such as III-nitrides (Nakamura and Krames 2013). However, in recent years, the field of optical cooling (Sheik-Bahae and Epstein 2007) witnessed exciting new developments, reviving the interest in III-arsenides (Olsson et al. 2016; Santhanam et al. 2012; Chen et al. 2017). Our recent work has provided first evidence that the threshold for electroluminescent cooling (ELC) can already be reached, at moderate and high powers and at $300 \mathrm{~K}$, locally in the LED within the intracavity double diode structure (DDS) (Olsson et al. 2016; Sadi et al. 2018; Radevici et al. 2018). The DDS incorporates a GaAs active region (AR) LED, optically coupled to a GaAs $\mathrm{p}-\mathrm{n}$ homojunction photodetector (PD), all within the same device. Reaching the ELC threshold and in particular making the effect accessible in technologically useful configurations will, however, require the minimization of several loss mechanisms and optimizing many aspects of the device. In particular, the impact of non-radiative recombination mechanisms at the interfaces and free surfaces of the LED active layer, and the PD detection losses should be reduced significantly (Olsson et al. 2016; Radevici et al. 2018; Sadi et al. 2018). In addition, there are also multiple related phenomena that will substantially affect the device performance and optimization, such as charge spreading and operation temperature. Therefore, it is interesting to compare e.g. the differences arising from using AlGaAs and GaInP barriers in the structure, as GaInP is known to allow substantially lower interface recombination velocities (Levinshtein et al. 1999), while introducing a large valence band offset that might have an effect on the hole transport over the interface. As acknowledged in literature, interface recombination is in general less important at GaInP/GaAs interfaces, as compared to AlGaAs/ GaAs interfaces (Levinshtein et al. 1999). As suggested in Refs. Aierken (2008), Sugino et al. (1998), Bednyi and Baidus (1996), III-P materials are characterized by a lower density of harmful interface states than III-As, corresponding to a lower density of deep-level (mid-gap) electronic traps, and hence a reduced net non-radiative recombination at the interfaces.

In this paper, we investigate the expected effects of interface recombination on the performance of a III-As intracavity DDS. In particular, we evaluate the impact of non-radiative interface recombination in the LED, by considering DDSs with LED structures based on either $\mathrm{GaInP} / \mathrm{GaAs}$ or $\mathrm{AlGaAs} / \mathrm{GaAs}$ double heterojunctions. For this purpose, we employ a calibrated electro-optical simulator, coupling the drift-diffusion (DD) formalism for electronic charge transport to a photon transport model, to show how better device designs can suppress the adverse effects of non-radiative recombination. This paper is organized as follows. In Sect. 2, we introduce the double diode structure and discuss the measurement techniques used for model calibration. In Sect. 3, we describe the simulation framework. In Sect. 4, we introduce selected figures of merit needed to analyze the DDS efficiency and ELC. In Sect. 5, we present results from the simulation of the aforementioned devices.

\section{The double diode structure}

Figure 1 illustrates the reference DDSs simulated in this work. They enclose double heterojunction (DHJ) GaInP/GaAs or AlGaAs/GaAs based LEDs on top of a GaAs light absorbing $\mathrm{p}-\mathrm{n}$ monojunction based $\mathrm{PD}$, all fabricated within the same semiconductor epistructure. 
(a)

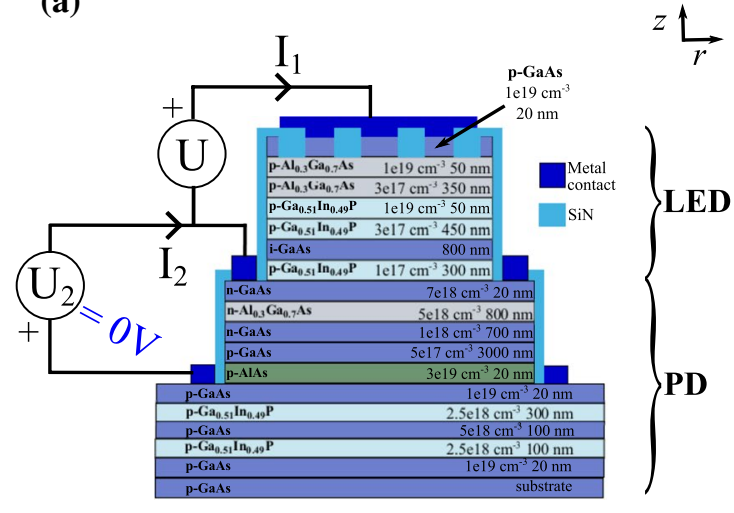

(b)

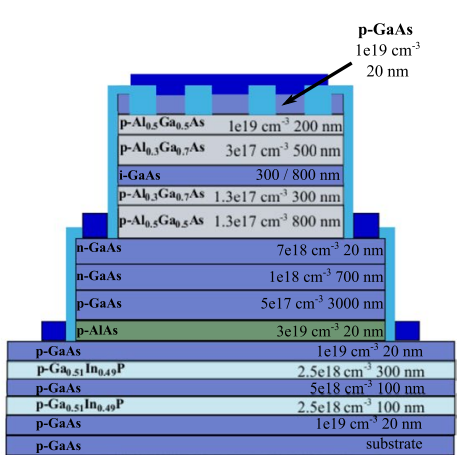

(c)

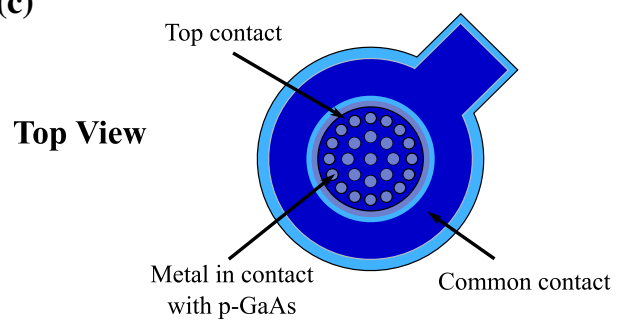

Fig. 1 The studied a GaInP/GaAs and b AlGaAs/GaAs intracavity DDSs, showing the layer arrangement including materials and doping concentrations (side view). c A top view of the full three-dimensional geometry. The top mesa diameter is $1 \mathrm{~mm}$

Figure 1 shows in detail the layer structure, sizes and doping concentrations, as well as the circular mesa arrangement. The LEDs for the GaInP/GaAs and AlGaAs/GaAs DDSs incorporate a 300 or $800 \mathrm{~nm}$ thick i-GaAs layer constituting the active region of the LED, while their PD p-n homojunction consists of relatively thick n-GaAs and p-GaAs layers (700 $\mathrm{nm}$ and $3 \mu \mathrm{m}$, respectively). The experimental data used to calibrate our simulator are obtained from measuring DDSs grown by both metalorganic vapour phase epitaxy and molecular beam epitaxy. The grown heterostructures were processed to define LED and PD mesas, as described in Refs. Olsson et al. (2016), Radevici et al. (2018). The simulated DDSs have a LED mesa diameter of $1 \mathrm{~mm}$.

In the DDSs, light emission from the LED is guided towards the PD, and the direct measurement of the LED and PD currents allows detecting the amount of absorbed light (Olsson et al. 2016). The devices are characterized electrically using three-point probe $I-V$ measurements, biasing the LED using an external voltage $U_{1}$ and measuring the current injected through the LED $I_{1}$, and the current generated in the short-circuited PD $I_{2}$ by the photons emitted by the LED (Olsson et al. 2016; Radevici et al. 2018). Both the LED and PD have a common ground contact as shown in Fig. 1. Hence, the DDS arrangement allows direct observation of the lower limit of energy transfer between LED and PD, quantitatively expressed with the coupling quantum efficiency (CQE) defined as the ratio $\eta_{C Q E}=I_{2} / I_{1}$, while circumventing the light extraction problems encountered in high-efficiency light emitter setups (Broell et al. 2014; Hurni et al. 2015; Gauck et al. 1997; Bender et al. 2013). The CQE is a central 
experimentally accessible figure of merit in the DDS context, as ELC can only be observed if the condition $P C E=\eta_{C Q E} \hbar \omega / U>1$ for the power conversion efficiency (PCE) is met. This condition indicates that the optical power emitted by the LED $I_{2} \hbar \omega / q$ is higher than electrical power injected to it $I_{1} U$. Here, $U$ is the LED bias, and $\hbar \omega$ is the energy of the emitted photons ( $\sim$ emitter bandgap $\left.E_{g}=1.42 \mathrm{eV}\right)$.

The DDS is a useful intermediate research device prototype that is expected to facilitate the direct demonstration of ELC. The incorporated LED structure in the DDS operates slightly differently in comparison to an isolated LED; for example, the integrated PD provides electron current to the LED and thereby facilitating current spreading in the LED (Radevici et al. 2018). Also, since the reduction of efficiency due to interface recombination competes with several other DDS factors, such as the extent of current spreading and carrier densities at the interface, it is more straightforward to understand the impact of interface recombination in the DDS, instead of an isolated LED. It allows the direct characterization of the device efficiency using straightforward $I-V$ measurements (three-point probe measurements) while minimizing significantly light extraction issues.

\section{Modelling methodology}

\subsection{Electronic charge transport model}

The DDSs, as shown in Fig. 1, are essentially cylindrically symmetric, and hence to minimize computational cost, the DD model is implemented in two-dimensions using the cylindrical coordinate system $(r, z)$, where $z$ is the vertical position and $r$ is the distance to the center of the DDS. We simulate electronic charge transport by solving self-consistently the two-dimensional DD equations (Sadi et al. 2018; Kivisaari et al. 2015):

$$
\begin{gathered}
\nabla \cdot(-\epsilon \nabla \phi)=\frac{\partial}{r \partial r}\left(-\epsilon r \frac{\partial \phi}{\partial r}\right)+\frac{\partial}{\partial z}\left(-\epsilon \frac{\partial \phi}{\partial z}\right)=q\left(p-n+N_{d}-N_{a}\right), \\
\nabla \cdot \mathbf{J}_{n}=\nabla \cdot\left(\mu_{n} n \nabla E_{f n}\right)=\frac{\partial}{r \partial r}\left(\mu_{n} n r \frac{\partial E_{f n}}{\partial r}\right)+\frac{\partial}{\partial z}\left(\mu_{n} n \frac{\partial E_{f n}}{\partial z}\right)=q(R-G), \\
\nabla \cdot \mathbf{J}_{p}=\nabla \cdot\left(\mu_{p} p \nabla E_{f p}\right)=\frac{\partial}{r \partial r}\left(\mu_{p} p r \frac{\partial E_{f p}}{\partial r}\right)+\frac{\partial}{\partial z}\left(\mu_{p} p \frac{\partial E_{f p}}{\partial z}\right)=-q(R-G),
\end{gathered}
$$

where $\phi$ is the electrostatic potential, $E_{f n}$ and $E_{f p}$ are the quasi-Fermi levels for electrons and holes, respectively, and $n$ and $p$ represent the electron and hole densities, respectively, which are calculated from the Fermi-Dirac distribution using $\phi$, and $E_{f n}$ and $E_{f p}$. Also, $\mathbf{J}_{n}$ and $\mathbf{J}_{p}$ are the electron and hole current densities, respectively, and $N_{d}$ and $N_{a}$ are the ionized donor and acceptor densities, respectively. Additionally, $\epsilon$ is the permittivity, and $\mu_{n}$ and $\mu_{p}$ are the electron and hole mobilities, respectively. $R(G)$ is the total recombination (generation) rate per unit volume. The recombination rates for Shockley-Read-Hall (SRH), radiative and Auger recombination processes $\left(R_{S R H}, R_{\text {rad }}\right.$ and $R_{\text {Aug }}$, respectively) are calculated using the well-known ABC parameterized formula (Kivisaari et al. 2015):

$$
R=R_{S R H}+R_{\text {rad }}+R_{\text {Aug }}=\left[\frac{A}{n+p+2 n_{i}}+B+C(n+p)\right]\left(n p-n_{i}^{2}\right) .
$$


Here, $n_{i}$ is the intrinsic carrier density. Also, $A, B$ and $C$ are the recombination constants for the SRH, radiative and Auger mechanisms, respectively. The rates for surface and interface non-radiative recombination are given by:

$$
R_{\text {surface }}=\left[\frac{v_{s r}}{n+p+2 n_{i}}\right]\left(n p-n_{i}^{2}\right),
$$

where $v_{s r}$ is the recombination velocity. Photon emission in the LED is coupled to absorption in the photodetector via the optical model described below. It is noteworthy that real devices are not perfectly symmetric, as assumed in our model, especially when including omnidirectional reflectors (ODRs), at the top LED $p$-contact, to minimize the optical reflection losses at the top-contact-GaAs system. From an electrical point of view, ODRs are located in a dense grid so that the current spreading will closely correspond to what would be achieved by a continuous contact, and that the perfect cylindrical symmetry of the structure is not significantly broken. Using ODRs are shown to improve the peak CQE by $\sim 5 \%$ from the fully symmetric structures (without ODRs), as discussed in Ref. Olsson et al. (2016), and should not affect the conclusions made about the characteristics and efficiency of the DDS.

\subsection{Optical transport model}

Electro-optical transport is modelled by coupling the total radiative recombination in the LED (i-GaAs) active region to the total generation in the PD (GaAs) layers via an optical coupling constant $\chi$, while using generation rates $G(r, z)$ assumed to follow the Beer-Lambert law within the PD (Sadi et al. 2018):

$$
G(r, z)=\alpha \chi e^{-\alpha z} \int R_{r a d}(r, z) \mathrm{d} z .
$$

Here, $\alpha$ is the optical absorption coefficient of the PD material (i.e. GaAs), whose value is set to $10^{4} \mathrm{~cm}^{-1}$ (Casey et al. 1975), and $R_{\text {rad }}$ is the radiative recombination rate in the LED active region. The constant $\chi$ includes mainly the effect of the optical reflection losses from the top mirror. It is calculated by employing multi-layered media models based on the dyadic Green's functions, as discussed in Refs. Sadi et al. (2013, 2014), determining the reflectivity of the top LED p-contact-cap-layer system. By using the refractive indices given in Ref. Olsson et al. (2016), $\chi$ is found to vary between $90 \%$ and $95 \%$ in the DDSs studied here. Using the computationally more affordable power attenuation description, given by Eq. (6), gives good agreement between experimental measurements and the presented model, as well as the model and more advanced optical simulations we have carried out using e.g. radiative transfer models (Oksanen and Tulkki 2011).

\subsection{Practical simulation considerations}

In general, the DD equation system is highly nonlinear, and being the workhorse of device technology computer aided design (TCAD) for decades, its solution schemes have attracted considerable interest [see e.g. Ghione and Benvenuti (1997)]. Our model has been implemented using the commercial simulation software COMSOL Multiphysics, with the equations being solved in the two-dimensional real space using finite-element meshing. For the linear part, we use the MUMPS sparse matrix direct solver, and the nonlinear iteration is 
performed using the Newton method with an automatically adjusting damping factor. The discretization is done using quadratic Lagrange functions. The meshing sizes along both axes have been chosen after a thorough sensitivity analysis is carried out. The meshing is non-uniform, with special attention given to areas near internal interfaces and external surfaces, where finer meshing is needed to model correctly any changes at these boundaries. While our implementation provides a satisfactory convergence for all the cases studied here, the solution process could possibly be further improved by using e.g. the ScharfetterGummel scheme (Scharfetter and Gummel 1969).

\section{Figures of merit}

As discussed earlier, the direct measurement of the DDS provides only a few meaningful figures of merit, including the CQE and PCE of the DDS. The latter quantity is the practical indicator of EL cooling in the LED, and reflects the combined efficiency of the LED and the PD. Device simulations, however, allow estimating several internal phenomena, providing access to additional figures of merit. These include e.g. the internal quantum efficiency (IQE) of the LED, which by definition is given by $\eta_{I Q E}^{L E D}=I_{\text {rad }} / I_{1}$, where $I_{\text {rad }}$ is the current associated with radiative recombination in the LED. The PCE of the LED, which we term the internal power conversion efficiency (IPCE), is related to the IQE via $\eta_{I P C E}=\eta_{I Q E}^{L E D} \hbar \omega / U$. An above-unity IPCE indicates internal cooling in the LED. It is also insightful to assign a separate figure of merit for the interface recombination in the DDS. The IQE of the LED can be factorized as a product of the overall efficiency $\eta_{0}$ and the interface recombination efficiency $\eta_{\text {int }}$ as

$$
\eta_{I Q E}^{L E D}=\frac{I_{\text {rad }}}{I_{1}}=\frac{I_{\text {rad }}+I_{\text {int }}}{I_{1}} \frac{I_{\text {rad }}}{I_{\text {rad }}+I_{\text {int }}}=\eta_{0} \eta_{i n t},
$$

where $I_{\text {int }}$ is the current generated through interface recombination.

\section{Results and discussion}

The simulation model is calibrated and validated using experimental data for the DDSs shown in Fig. 1, obtained from the three-point probe measurement technique (Olsson et al. 2016; Radevici et al. 2018) in the form of $J-V$ and CQE curves. The data are extracted using pulsed $J-V$ measurements, suppressing almost completely device self-heating. This is well in line with the aim of this work, focusing on understanding how the materials and device geometry affect the CQE. Therefore, in the presented results, we assume that the DDSs have an approximately constant and homogeneous temperature $(300 \mathrm{~K})$ and therefore do not include thermal models in the simulations. Such an approximation is reasonable considering the fact that the actual DDSs have a fairly large thermal conductivity and are not (at present) meant to produce significant temperature differences. For both the DDS types, we use the established values for the SRH $\left[A \sim 3 \times 10^{5} \mathrm{~s}^{-1}\right.$ (Gilliland et al. 1993)], radiative $\left[B \sim 2 \times 10^{-10} \mathrm{~cm}^{3} \mathrm{~s}^{-1}\right.$ (Olsson et al. 2016) $]$ and Auger $\left[C \sim 10^{-30} \mathrm{~cm}^{6} \mathrm{~s}^{-1}\right.$ (Olsson et al. 2016)] recombination constants. The values of the surface $V_{s r}$ and interface $V_{\text {int }}$ recombination velocities are calibrated against the experiments. For the GaAs/air free surfaces at the edge of the LED mesa, a recombination velocity $V_{s r} \sim 4 \times 10^{5} \mathrm{~cm} / \mathrm{s}$ [close to values reported in literature (Levinshtein et al. 1999; Pavesi and Guzzi 1994)] is used, as it 
provides a perfect fit for the exponential part of the LED $J_{1}-V$ curve, at low biases. Also, a recombination velocity $V_{\text {int }} \sim 1.5 \mathrm{~cm} / \mathrm{s}$ is used at GaInP/GaAs interfaces [also within the range reported in literature (Levinshtein et al. 1999)]. For the AlGaAs/GaAs interfaces, a value of $\sim 450 \mathrm{~cm} / \mathrm{s}$ yields the best agreement with experiments. This value is also well within the range of values reported in the literature (Gilliland et al. 1993; Pavesi and Guzzi 1994; Reich et al. 1999).

Figure 2 illustrates the general characteristics and the excellent agreement between the calibrated simulations and measurements, with Fig. 2a showing the $J-V$ characteristics of the LED and PD for the GaInP/GaAs based DDS, and Fig. 2b showing the CQE for both the $\mathrm{GaInP} / \mathrm{GaAs}$ and $\mathrm{AlGaAs} / \mathrm{GaAs}$ based DDSs. We start the analysis by estimating the ideality factor $\gamma$, an important electrical property of diodes (David et al. 2016), from the linear (low bias) region of the $J-V$ curves as Olsson et al. (2016): $\gamma=\frac{q}{k_{b} T}\left[\frac{\mathrm{d} \ln J}{\mathrm{~d} V}\right]^{-1}$. Here, $q$ is the elementary charge, $k_{b}$ is the Boltzmann constant, and $T$ is the ambient temperature $(300 \mathrm{~K})$. Figure $2 \mathrm{a}$ indicates an ideality factor of $\sim 2$ for the LED at low biases $(<\sim 1 \mathrm{~V})$, where non-radiative surface recombination is the main current driving mechanism. The PD current-voltage curve further shows that radiative recombination in the LED is of the bimolecular form with an ideality factor $\sim 1$, at least up to the regime where the device enters the resistive limited operation region. Figure $2 b$ shows the CQE, as a function of LED bias, for both the GaInP/GaAs and AlGaAs/GaAs based DDSs, with peak values of $70 \%$ and $50 \%$, respectively. In general, the $\mathrm{AlGaAs} / \mathrm{GaAs}$ device gives a significantly lower $\mathrm{CQE}$, for all the biasing range, due to the increased role of recombination at the interfaces of the AlGaAs/GaAs/AlGaAs DHJ near the AR, as more thoroughly discussed below.

Figure 3 shows the conduction and valence band edges as well as the quasi-Fermi levels for electrons and holes, for various low and high biases: $0.2 \mathrm{~V}, 0.6 \mathrm{~V}, 1 \mathrm{~V}$ and $1.3 \mathrm{~V}$. As can be seen, the quasi-Fermi levels for electrons and holes within the LED split as soon as a bias is applied. However, the quasi-Fermi levels in the photodetector only start to split, in a visible fashion, at biases above $0.3 \mathrm{~V}$ when a sufficient photogenerated current is present.

Fig. 2 a The experimental and simulated LED $\left(J_{1}-U\right)$ and photodetector $\left(J_{2}-U\right)$ characteristics, for the GaInP/ GaAs DDS illustrated in Fig. 1. b The experimental and simulated CQE as a function of LED bias, for both the GaInP/GaAs and $\mathrm{AlGaAs} / \mathrm{GaAs}(\mathrm{AR}=300 \mathrm{~nm})$ based DDSs illustrated in Fig. 1
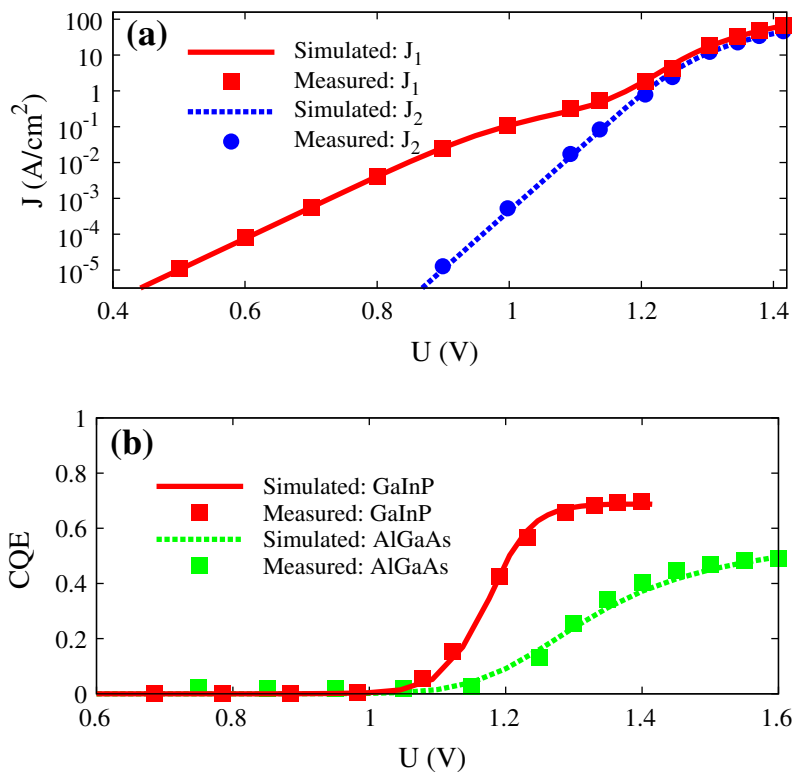

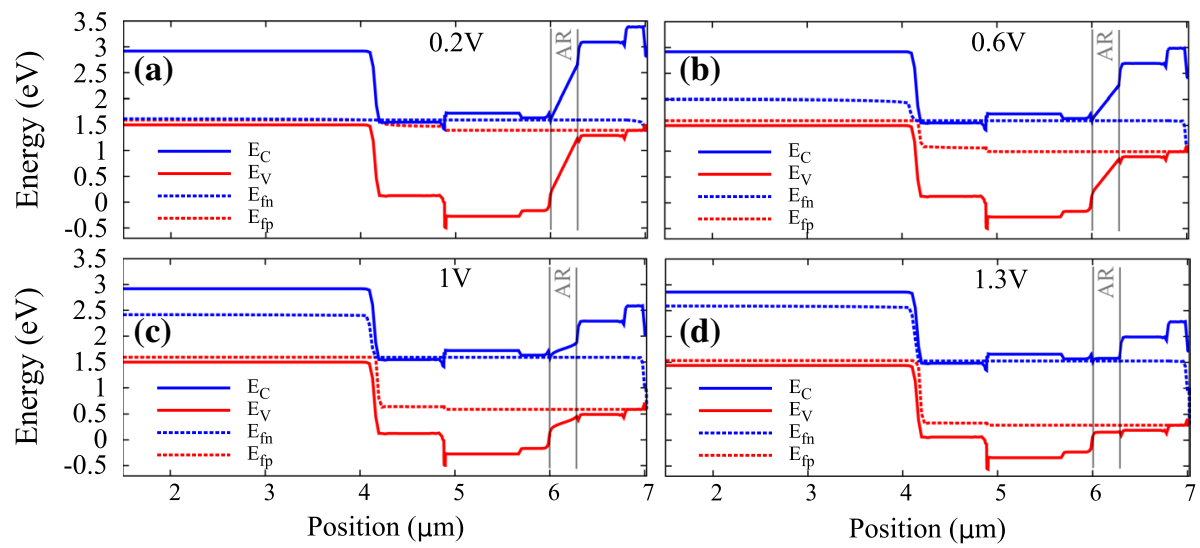

Fig. 3 Conduction and valence band edges as well as the quasi-Fermi levels for electrons and holes, for various low and moderate injection biases: a $0.2 \mathrm{~V}, \mathbf{b} 0.6 \mathrm{~V}, \mathbf{c} 1 \mathrm{~V}$ and d $1.3 \mathrm{~V}$

The split becomes more visible at stronger injection (e.g. 1.3 V) where LED photon emission results in the generation of a significant number of electron-hole pairs, giving rise to a significant PD current. Close inspection of the Fermi levels and band edges at high biases $(1.3 \mathrm{~V})$ suggests that only the two ( $\mathrm{AlGaAs} / \mathrm{GaAs}$ high recombination velocity) interfaces limiting the active region, as highlighted in Fig. 3, are characterized by a simultaneously (relatively) high electron an hole densities. Indeed, further analysis (not shown here) indicates that almost all of the non-radiative interface recombination events occur at these two interfaces, and that this type of recombination is negligible at all the other interfaces. The same conclusions hold for the GaInP/GaAs DDS. Specifically, the electron density at the $\mathrm{p}-\mathrm{GaInP} / \mathrm{p}-\mathrm{AlGaAs}$ interface of the LED is negligible (calculated to be several orders of magnitude lower than the hole density), yielding insignificant interface recombination rates.

To illustrate the effect the choice of the barrier material has on the key figures of merit, we show in Fig. 4 both the DDS PCE and LED internal PCE (IPCE), as a function of bias. The IPCE corresponds to the internal efficiency of the LED, without considering e.g. the photodetector losses that may be eliminated also in practice, e.g. by optimizing the layer

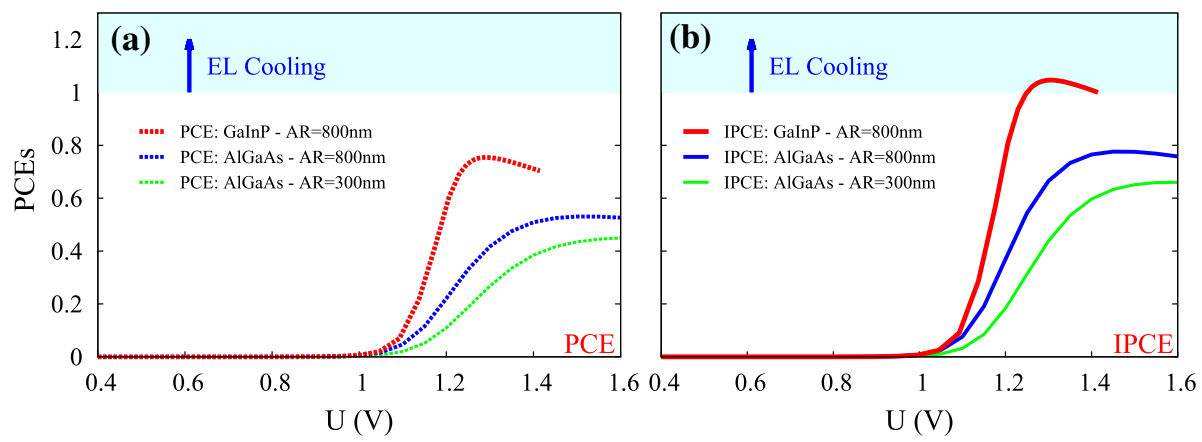

Fig. 4 The simulated PCE and IPCE, as a function of LED bias, for both (i) the GaInP/GaAs DDS and (ii) the AlGaAs/GaAs DDS with AR thicknesses of $300 \mathrm{~nm}$ and $800 \mathrm{~nm}$ 
structure of the PD. The PCE of both devices remains clearly below unity. For the GaInP/ GaAs device, this is primarily due to inefficient photodetection. For the AlGaAs/GaAs device, this is also due to interface recombination which introduces another significant loss source. Interestingly, the peak PCE of the $\mathrm{GaInP} / \mathrm{GaAs}$ device takes place at a much lower voltage than that of the $\mathrm{AlGaAs} / \mathrm{GaAs}$ device. Figure 4b, however, shows that the IPCE of the LED already exceeds unity for the GaInP/GaAs DDS at high biases, while the IPCE of the $\mathrm{AlGaAs} / \mathrm{GaAs}$ based device peaks at $\sim 0.8$. This indicates that the bulk properties of the GaInP/GaAs structures are sufficient to reach internal cooling in the LED. The superior performance of the GaInP/GaAs structure is enabled by the low $A$ in the high quality GaAs active layer, but also the very low recombination velocity $(\sim 1.5 \mathrm{~cm} / \mathrm{s})$ at the $\mathrm{GaInP} / \mathrm{GaAs}$ interfaces (Levinshtein et al. 1999). Figure 4 additionally shows the PCEs for AlGaAs/ GaAs DDSs with an AR thickness of $300 \mathrm{~nm}$, in addition to the $800 \mathrm{~nm}$ device, illustrating how the role of interface recombination further increases as the AR thickness is reduced. In general, the AlGaAs/GaAs DDSs exhibit much lower PCEs, which is mainly expected to be due to the increased recombination velocity $(\sim 450 \mathrm{~cm} / \mathrm{s})$ at the $\mathrm{AlGaAs} / \mathrm{GaAs}$ interfaces of the AR (Levinshtein et al. 1999; Pavesi and Guzzi 1994; Reich et al. 1999). Specifically, the AlGaAs/GaAs DDS with the thinner AR $(300 \mathrm{~nm})$ gives the worst performance, due to the increased interface-area-to-AR-volume ratio. As the AR thickness is increased to $800 \mathrm{~nm}$, the efficiency is improved visibly with the peak PCE and IPCE increasing from $45 \%$ and $66 \%$ to $53 \%$ and $78 \%$, respectively.

To quantify the interface recombination losses, we show in Fig. 5a the interface recombination efficiency $\eta_{i n t}$ as a function of the LED bias. As expected, interface recombination plays a very negligible role in $\mathrm{GaInP} / \mathrm{GaAs}$ structures, as indicated by the near-unity $\eta_{\text {int }}$ in this device throughout the biasing range. On the other hand, the losses associated with interface recombination for the AlGaAs/GaAs DDSs are extensive and dominate over the

Fig. 5 a The dependence of $\eta_{\text {int }}$, as a function of LED bias, for both (i) the GaInP/GaAs DDS and (ii) the AlGaAs/GaAs DDS with AR thicknesses of $300 \mathrm{~nm}$ and $800 \mathrm{~nm}$. b The ratios of the Auger and leakage currents in the LED to the total LED current, as a function of bias, for the GaInP/ GaAs DDS and the AlGaAs/ GaAs DDS with an AR thickness of $300 \mathrm{~nm}$
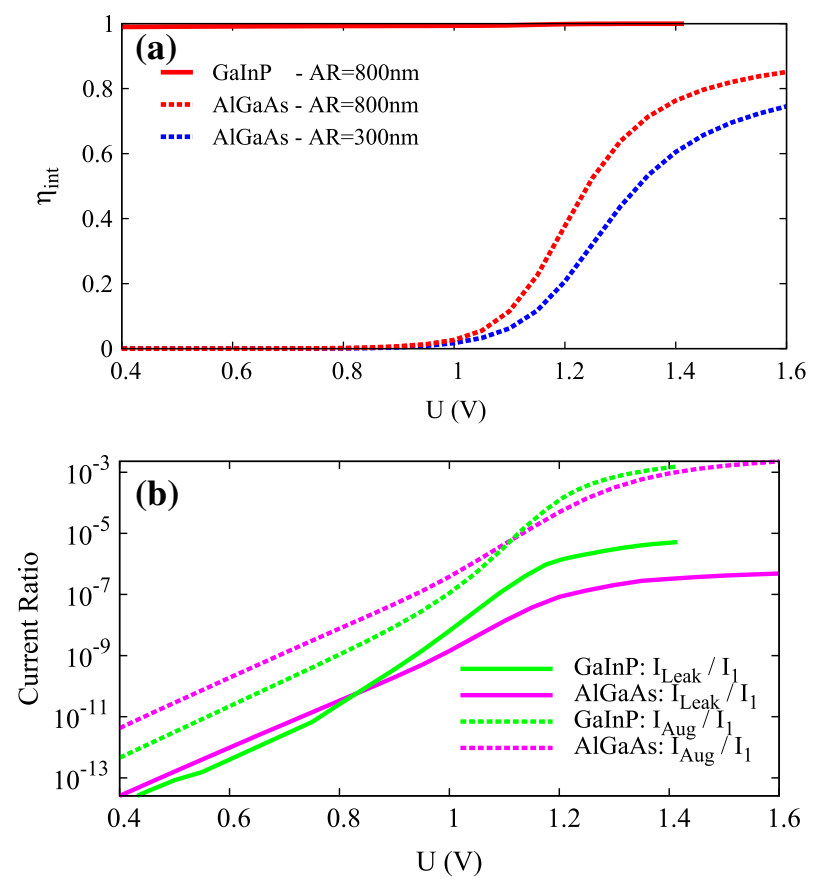
radiative recombination at biases up to $1 \mathrm{~V}$. At larger biases, where radiative recombination becomes stronger, its impact is reduced but the peak value of $\eta_{i n t}$ still remains so low, at 0.85 , that it seems unlikely that AlGaAs barriers could allow reaching the ELC threshold, unlike the GaInP barriers. As expected, $\eta_{\text {int }}$ is lower when using the (thinner) $300 \mathrm{~nm}$ thick AR, peaking at 0.73 , as a higher interface-area-to-AR-volume ratio favours interface recombination over bulk processes, such as radiative recombination. Figure $5 \mathrm{~b}$ shows the ratios of the Auger and leakage currents in the LED to the total LED current, as a function of bias, for the GaInP/GaAs DDS and the AlGaAs/GaAs DDS with an AR thickness of $300 \mathrm{~nm}$. Auger recombination is negligible in the studied GaAs based LEDs, for the biases considered here as illustrated in Fig. 5b, considering the relatively low Auger constant of bulk GaAs (Strauss et al. 1993). The minority carrier leakage is also negligible for the normal operating biases.

\section{Conclusions}

To conclude, we couple the DD formalism with an optical model to compare the effect of GaInP and AlGaAs barriers on interface recombination and the efficiency of III-As double diode structures, designed to demonstrate electroluminescent cooling, at high biases, in LEDs with GaAs active regions. The modelling framework is validated with experiments, and is used to show that using GaInP/GaAs DHJs allows the power conversion efficiency of the LED to exceed unity, corresponding to electroluminescent cooling. This observation indicates that the bulk properties of the DDS materials are sufficient for LED cooling. However, for AlGaAs/GaAs DHJ based DDSs, the predicted larger interface recombination velocities may represent a serious challenge for observing cooling.

Acknowledgements Open access funding provided by Aalto University. We acknowledge funding from the Academy of Finland and the European Research Council under the Horizon 2020 research and innovation programme (Grant Agreement No 638173).

Open Access This article is distributed under the terms of the Creative Commons Attribution 4.0 International License (http://creativecommons.org/licenses/by/4.0/), which permits unrestricted use, distribution, and reproduction in any medium, provided you give appropriate credit to the original author(s) and the source, provide a link to the Creative Commons license, and indicate if changes were made.

\section{References}

Aierken, A.: Passivation of GaAs surfaces and fabrication of self-assembled $\operatorname{In}(\mathrm{Ga}) \mathrm{As} / \mathrm{GaAs}$ quantum ring structures. Ph.D. dissertation, Aalto University (2008)

Bednyi, B.I., Baidus, N.V.: Passivation of a GaAs surface by treatment in phosphine vapor. Semiconductors 30, 132-135 (1996)

Bender, D.A., Cederberg, J.G., Wang, C., Sheik-Bahae, M.: Development of high quantum efficiency GaAs/ GaInP double heterostructures for laser cooling. Appl. Phys. Lett. 102, 252102 (2013)

Broell, M., Sundgren, P., Rudolph, A., Schmid, W., Vogl, A., Behringer, M.: New developments on highefficiency infrared and InGaAlP light-emitting diodes at OSRAM opto semiconductors. IN: LightEmitting Diodes: Materials, Devices, and Applications for Solid State Lighting XVIII vol. 9003, p. 90030L-1-6 (2014)

Casey, H.C., Sell, D.D., Wecht, K.W.: Concentration dependence of the absorption coefficient for n- and p-type GaAs between 1.3 and 1.6 eV. J. Appl. Phys. 46, 250-257 (1975) 
Chen, K., Xiao, T.P., Santhanam, P., Yablonovitch, E., Fan, S.: High-performance near-field electroluminescent refrigeration device consisting of a GaAs light emitting diode and a Si photovoltaic cell. J. Appl. Phys. 122, 143104 (2017)

David, A., Hurni, C.A., Young, N.G., Craven, M.D.: Electrical properties of III-Nitride LEDs: recombination-based injection model and theoretical limits to electrical efficiency and electroluminescent cooling. Appl. Phys. Lett. 109, 083501 (2016)

Gauck, H., Gfroerer, T.H., Renn, M.J., Cornell, E.A., Bertness, K.A.: External radiative quantum efficiency of $96 \%$ from a GaAs / GaInP heterostructure. Appl. Phys. A: Mater. Sci. Process. 64, 143-147 (1997)

Ghione, G., Benvenuti, A.: Discretization Schemes for high-frequency semiconductor device models. IEEE Trans. Antennas Propag. 45, 443-456 (1997)

Gilliland, G.D., Wolford, D.J., Kuech, T.F., et al.: Minority-carrier recombination kinetics and transport in surface-free GaAs/Al ${ }_{x} \mathrm{Ga}_{1-x}$ As double heterostructures. J. Appl. Phys. 73, 8386-8396 (1993)

Hurni, C.A., David, A., Cich, M.J., Aldaz, R.I., Ellis, B., Huang, K., Tyagi, A., DeLille, R.A., Craven, M.D., Steranka, F.M., Krames, M.R.: Bulk GaN flip-chip violet light-emitting diodes with optimized efficiency for high-power operation. Appl. Phys. Lett. 106, 031101 (2015)

Kivisaari, P., Oksanen, J., Tulkki, J., Sadi, T.: Monte Carlo simulation of hot carrier transport in III-N LEDs. J. Comput. Electron. 14, 382-397 (2015)

Levinshtein, M., Rumyantsev, S., Shur, M.: [Ternary And Quaternary III-V Compounds], Handbook Series on Semiconductor Parameters. World Scientific Publishing, Singapore (1999)

Nakamura, S., Krames, M.R.: History of gallium-nitride-based light-emitting diodes for illumination. Proc. IEEE 101, 2211-2220 (2013)

Oksanen, J., Tulkki, J.: Effects of photon transport, emission saturation and reflection losses on thermophotonic cooling. Proc. SPIE 7951, 79510H (2011)

Olsson, A., Tiira, J., Partanen, M., et al.: Optical energy transfer and loss mechanisms in coupled intracavity light emitters. IEEE Trans. Elect. Devices 63, 3567-3573 (2016)

Pavesi, L., Guzzi, M.: Photoluminescence of $\mathrm{Al}_{x} \mathrm{Ga}_{1-x}$ As alloys. J. Appl. Phys. 75, 4779-4842 (1994)

Radevici, I., Tiira, J., Sadi, T., Oksanen, J.: Influence of photo-generated carriers on current spreading in double diode structures for electroluminescent cooling. Semicond. Sci. Technol. 33, 05LT01 (2018)

Reich, I., Diaz, P., Prutskij, T., Mendoza, J., Vargas, H., Marin, E.: Photoacoustic determination of the recombination velocity at the AlGaAs/GaAs heterostructure interface. J. Appl. Phys. 86, 6222 (1999)

Sadi, T., Radevici, I., Kivisaari, P., Casado, A., Oksanen, J.: Electroluminescent cooling using double diode structures. In: Proceedings of 18th International Conference on Numerical Simulation of Optoelectonic Devices (NUSOD’18), pp. 125-126 (2018)

Sadi, T., Oksanen, J., Tulkki, J.: Effect of plasmonic losses on light emission enhancement in quantum-wells coupled to metallic gratings. J. Appl. Phys. 114, 223104 (2013)

Sadi, T., Oksanen, J., Tulkki, J.: Improving light extraction from GaN light-emitting diodes by buried nanogratings. IEEE J. Quantum Electron. 50, 141-147 (2014)

Sadi, T., Kivisaari, P., Tiira, J., Radevici, I., Haggren, T., Oksanen, J.: Electroluminescent cooling in intracavity light emitters: modeling and experiments. Opt. Quantum Electron. 50, 18 (2018)

Santhanam, P., Gray, D.J., Ram, R.J.: Thermoelectrically pumped light-emitting diodes operating above unity efficiency. Phys. Rev. Lett. 108, 097403 (2012)

Scharfetter, D.L., Gummel, H.K.: Large-signal analysis of a silicon read diode oscillator. IEEE Trans. Electron. Devices 16, 64-77 (1969)

Sheik-Bahae, M., Epstein, R.I.: Optical refrigeration. Nat Photonics 1, 693-699 (2007)

Strauss, U., Rühle, W.W., Köhler, K.: Auger recombination in intrinsic GaAs. Appl. Phys. Lett. 62, 55-57 (1993)

Sugino, T., Ninomiya, H., Shirafuji, J., Matsuda, K.: Characterization of GaAs surfaces treated with phosphine gas photodecomposed by an ArF excimer laser. Appl. Phys. Lett. 72, 1472-1474 (1998)

Publisher's Note Springer Nature remains neutral with regard to jurisdictional claims in published maps and institutional affiliations. 